\title{
Multi-frequency VLBI observations of NRAO 530
}

\author{
S.-W. Feng ${ }^{1,2}$, Z.-Q. Shen ${ }^{1,3}$, H.-B. Cai ${ }^{1,2}$, X. Chen ${ }^{1,2}$, R.-S. Lu ${ }^{1,2}$, and L. Huang ${ }^{1,2}$ \\ 1 Shanghai Astronomical Observatory, Chinese Academy of Sciences, Shanghai 200030, PR China \\ e-mail: winfeng@shao.ac.cn \\ 2 Graduate School of Chinese Academy of Sciences, Beijing 100012, PR China \\ 3 Joint Institute for Galaxy and Cosmology, SHAO and USTC, PR China
}

Received 12 December 2005 / Accepted 2 May 2006

\section{ABSTRACT}

\begin{abstract}
We report on VLBA observations of a $\gamma$-ray bright blazar NRAO 530 at multiple frequencies $(5,8,15,22,39,43$, and $45 \mathrm{GHz})$ in 1997 and 1999. These multi-epoch multi-frequency high-resolution VLBI images exhibit a consistent core-dominated morphology with a bending jet to the north of the core. The quasi-simultaneous data observed at five frequencies $(5,8,15,22$, and $43 \mathrm{GHz})$ in February 1997 enabled us to estimate the spectra of compact VLBI components in this highly variable source. Flat spectra are seen in the two central components (A and B), and the most compact component $\mathrm{A}$ that has the flattest spectral index at the south end is identified as the core. Based on the synchrotron cooling timescale argument, it is suggested that the observed inverted spectrum of component $\mathrm{C}$ is caused by the free-free absorption (FFA), although the synchrotron self-absorption (SSA) model cannot be ruled out definitely. While the SSA probably exists in component B, it is likely that the same FFA would produce the spectral turnover toward component B since the fitted FFA coefficients in both B and C components are almost the same. If so, the projected size of such an absorbing medium is at least $25 \mathrm{pc}$.

By adding our new measurements to previous data, we obtain apparent velocities of two components, B of $10.2 \mathrm{c}$ and $\mathrm{E}$ of $14.5 \mathrm{c}$. These are consistent with the emergence of VLBI component associated with the flux density outburst; i.e. components B and E are related to strong $\gamma$-ray flares in 1994.2-1994.6 and 1995.4-1995.5, respectively. We investigate the spectral variability further by making use of the single-dish measurements covering a complete outburst profile from mid-1994 to mid-1998. It shows a continuous increase in the turnover frequency during the rising phase, and a gradual decrease after passing the peak of the flare. Finally, we discuss the equipartition Doppler-factor $\left(\delta_{\mathrm{eq}}\right)$ based on analysis of the magnetic field and obtain $\delta_{\mathrm{eq}} \mathrm{s}$ of 3.7, 7.2, and 0.8 for components $\mathrm{A}$, $\mathrm{B}$, and $\mathrm{C}$, respectively, which are all consistent with a larger flux density in component $\mathrm{B}$, the non-detection of proper motion in component $\mathrm{C}$, and a bent jet.
\end{abstract}

Key words. galaxies: quasars: individual: NRAO 530 - galaxies: jets - radio continuum: galaxies

\section{Introduction}

NRAO 530 (PKS 1730-130) is an 18.5 mag QSO (Welch \& Spinrad 1973) with a redshift $z=0.902$ (Junkkarinen 1984). It is variable at radio wavelengths (Medd et al. 1972; Marscher et al. 1979 ) and is an X-ray emitter (Marscher \& Broderick 1981). On the basis of its optical variability (Pollock et al. 1979), NRAO 530 is also classified as one of the optically violent variables (OVVs).

Figure 1 (top) shows the flux-density history at 4.8, 8.0, and $14.5 \mathrm{GHz}$ monitored by the University of Michigan Radio Astronomy Observatory (cf. Aller et al. 1985). It can be seen that flux density changed a lot from 1994.5 to 1998.5. NRAO 530 underwent a dramatic millimeter flare beginning in 1994, when two distinct flares nearly tripled the $90 \mathrm{GHz}$ flux (Bower et al. 1997). EGRET reported increasing $\gamma$-ray flux from the direction of NRAO 530 between 1991 and 1995 (Hartman et al. 1999). To investigate the relationship between these flares (at radio and $\gamma$ ray) and the possible emergence of new component, we present multi-epoch and multi-frequency VLBI study of NRAO 530. We discuss the kinematics of the jet components, the possible absorption mechanism, and the spectral variability.

Throughout this paper we adopt the cosmological parameters $H_{0}=71 \mathrm{~km} \mathrm{~s}^{-1} \mathrm{Mpc}^{-1}, \Omega_{\mathrm{M}}=0.27$, and $\Omega_{\Lambda}=0.73$. This results in a luminosity distance to NRAO 530 of $D_{\mathrm{L}}=5.8 \mathrm{Gpc}$ and an angular-to-linear size conversion factor of $7.8 \mathrm{pc} \mathrm{mas}^{-1}$. The spectral index $\alpha$ is defined by $S_{v} \propto v^{-\alpha}$.

\section{VLBI observations and data reduction}

NRAO 530 was observed at 5, 8, 15, 22, and $43 \mathrm{GHz}$ in February 1997 with the VLBA plus a VLA antenna. It was also observed 3 times in 1999 at 39, 43, and $45 \mathrm{GHz}$ with the VLBA. Detailed parameters of observations can be found in Table 1. The data were recorded in VLBA format with a total bandwidth of $32 \mathrm{MHz}$ (4 IFs) for left circular polarization (LCP) (and also right circular polarization (RCP) in 1997). The observed data were cross-correlated with the VLBA correlator at Socorro, New Mexico of the USA.

The post-correlation data reduction was carried out using the NRAO AIPS and Caltech DIFMAP packages. We first applied amplitude calibration (atmospheric opacity corrections were made at frequencies $\geq 22 \mathrm{GHz}$ ) using the gain curves and system temperatures measured at each station. Then fringe fitting was performed to determine the instrumental delays first, and then to solve for the residual delays, rates, and phases. The data were then exported into DIFMAP for self-calibration imaging. These visibilities were time-averaged into 30-s bins with uncertainties estimated from the scatter of data points within the $30 \mathrm{~s}$ interval. The data were inspected for obviously bad points, 
Table 1. VLBI observations of NRAO 530.

\begin{tabular}{ccccccc}
\hline \hline $\begin{array}{c}v \\
(\mathrm{GHz})\end{array}$ & $\begin{array}{c}\text { Epoch } \\
(\text { year }) \\
(1)\end{array}$ & $\begin{array}{c}\text { Integration time } \\
(\mathrm{min}) \\
(3)\end{array}$ & $\begin{array}{c}\text { Bandwidth } \\
(\mathrm{MHz}) \\
(4)\end{array}$ & $\begin{array}{c}\text { Bit rate } \\
(\mathrm{bit}) \\
(5)\end{array}$ & Polarization(s) & Antennas \\
\hline 5.0 & 1997.10 & 20 & $32 \times 2$ & 1 & RCP, LCP & VLBA+VLA1 \\
8.4 & 1997.10 & 20 & $32 \times 2$ & 1 & RCP, LCP & VLBA+VLA1 \\
15.4 & 1997.12 & 20 & $32 \times 2$ & 1 & RCP, LCP & VLBA+VLA1 \\
22.2 & 1997.12 & 20 & $32 \times 2$ & 1 & RCP, LCP & VLBA+VLA1 \\
43.2 & 1997.12 & 20 & $32 \times 2$ & 2 & RCP, LCP & VLBA+VLA1 \\
43.1 & 1999.32 & 17 & 32 & 2 & LCP & VLBA \\
43.1 & 1999.40 & 17 & 32 & 2 & LCP & VLBA \\
39.1 & 1999.42 & 19 & 32 & 2 & LCP & VLBA \\
45.1 & 1999.42 & 18 & 32 & 2 & LCP & VLBA \\
\hline
\end{tabular}

Notes: (1) Observing frequency in GHz; (2) observing epoch in yr; (3) total on-source time in minutes; (4) recording bandwidth in MHz; (5) sampling rate in bit; (6) polarization mode, and (7) observing antennas.
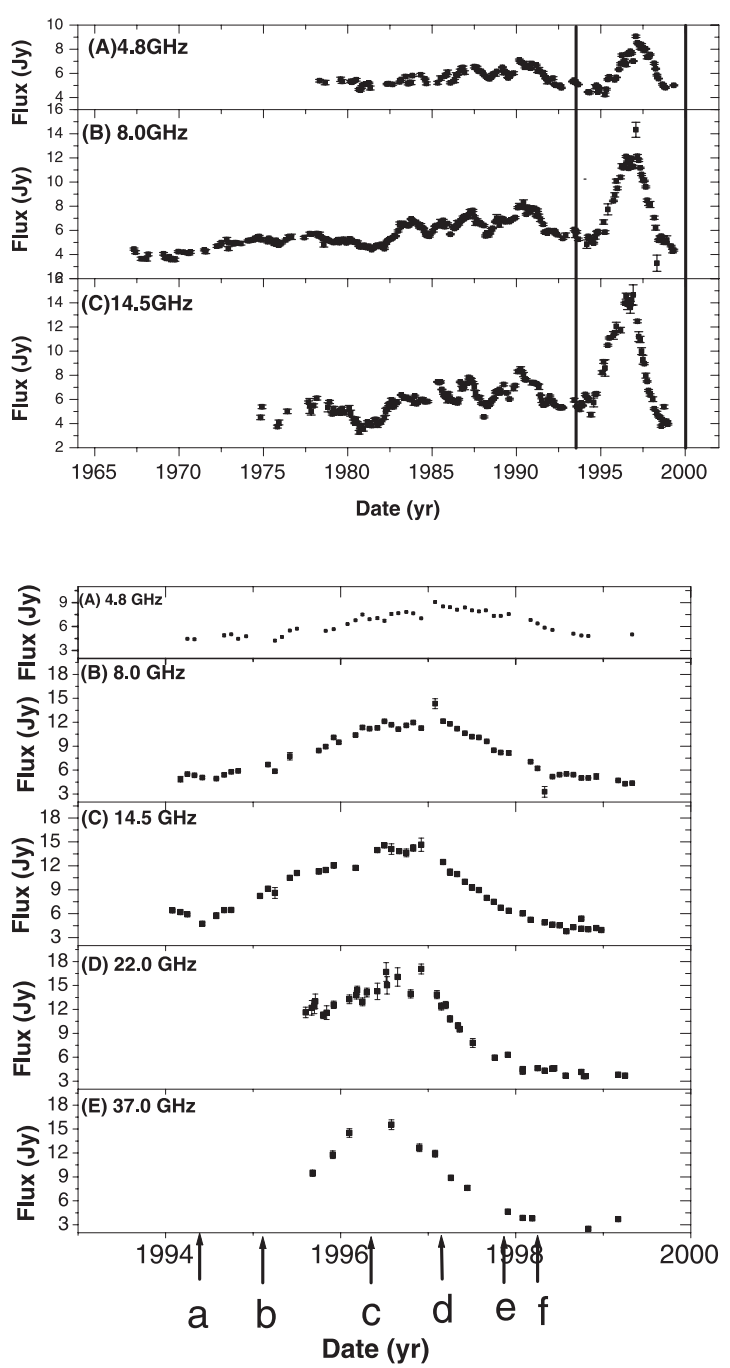

Fig. 1. Top: light curves of NRAO 530 at (A) $4.8 \mathrm{GHz}$, (B) $8.0 \mathrm{GHz}$, (C) $14.5 \mathrm{GHz}$ from UMRAO (University of Michigan Radio Astronomy Observatory). Bottom: light curves of NRAO 530 observed during the period from 1994 to 1999 at (A) $4.8 \mathrm{GHz}$, (B) $8.0 \mathrm{GHz}$, (C) $15.4 \mathrm{GHz}$ from UMRAO and (D) $22 \mathrm{GHz}$, (E) $37 \mathrm{GHz}$ from 13.7-meter antenna at Metsahovi Radio Observatory (Teräsranta et al. 2004), arrows with letters a, b, c, d, e, and f indicate different epochs plotted in Fig. 6.

most of which were near the beginning of the scan when telescopes were still slewing to the sources. Images were made using the self-calibration and clean procedure for several iterations. A 1-Jy point-source model was employed at the start of each mapping process and the natural weighting of the data was used for all nine images shown in Fig. 2. The detailed paraments of images are summarized in Table 2. For a quantitative analysis of the structure in these images, we made model fits to the calibrated visibility data with elliptical Gaussian components. The results are listed in Table 3. A maximum uncertainty of $15 \%$ in the flux density was estimated from the uncertainties of the amplitude calibration and the formal errors of the model fits. The typical uncertainties of the component positions and their sizes were examined to be about $10 \%$ of the fitted values. The brightness temperature (Col. 11 in Table 3) of each component was calculated using the following equation (i.e. Shen et al. 1997),

$T_{\mathrm{b}}=1.22 \times 10^{12} S v^{-2}\left(\theta_{\text {maj }} \theta_{\text {min }}\right)^{-1}(1+z) K$,

where $S$ is the flux density in Jy, $v$ the frequency in $\mathrm{GHz}, z$ the redshift, $\theta_{\text {maj }}$ and $\theta_{\text {min }}$ the sizes (FWHM) of the major and minor axes in mas, respectively.

\section{Results}

\subsection{Components and their proper motion}

The source shows different morphologies at different frequencies with more details revealed at higher frequencies in the core region (see Fig. 2). The overall structure is dominated by a compact core plus a jet-like feature extending approximately 5 mas from the core along a position angle (PA) of $15^{\circ}$ that further bends toward the north. Figure 3 is the projected trajectory based on the locations of the VLBI components in NRAO 530 observed in February 1997, inferring a continuously bending path of the jet emission. Here, we assume the brightest and most compact component A (see Table 3 ) as the core, because it is located at the south end of emission and has the flattest spectral index (see Sect. 3.2). The same core identification is also adopted by Jorstad et al. (2001).

Component $\mathrm{D}$ at about 22-23 mas north of the core region was detected at 5 and $8 \mathrm{GHz}$ (Figs. $2 \mathrm{a}$ and b). This is probably associated with the knot emission at about 25 mas north of the core and seen from the $1.7 \mathrm{GHz}$ VLBI observations (Bondi et al. 1996). At about 3-4 mas from the core with a PA of $10-15^{\circ}$, component $\mathrm{C}$ was consistently detected at frequencies of 5,8 , 15, and $22 \mathrm{GHz}$ (Figs. 2a-d). This component has also been seen by Tingay et al. (1998), Shen et al. (1997), and Jorstad et al. (2001). 

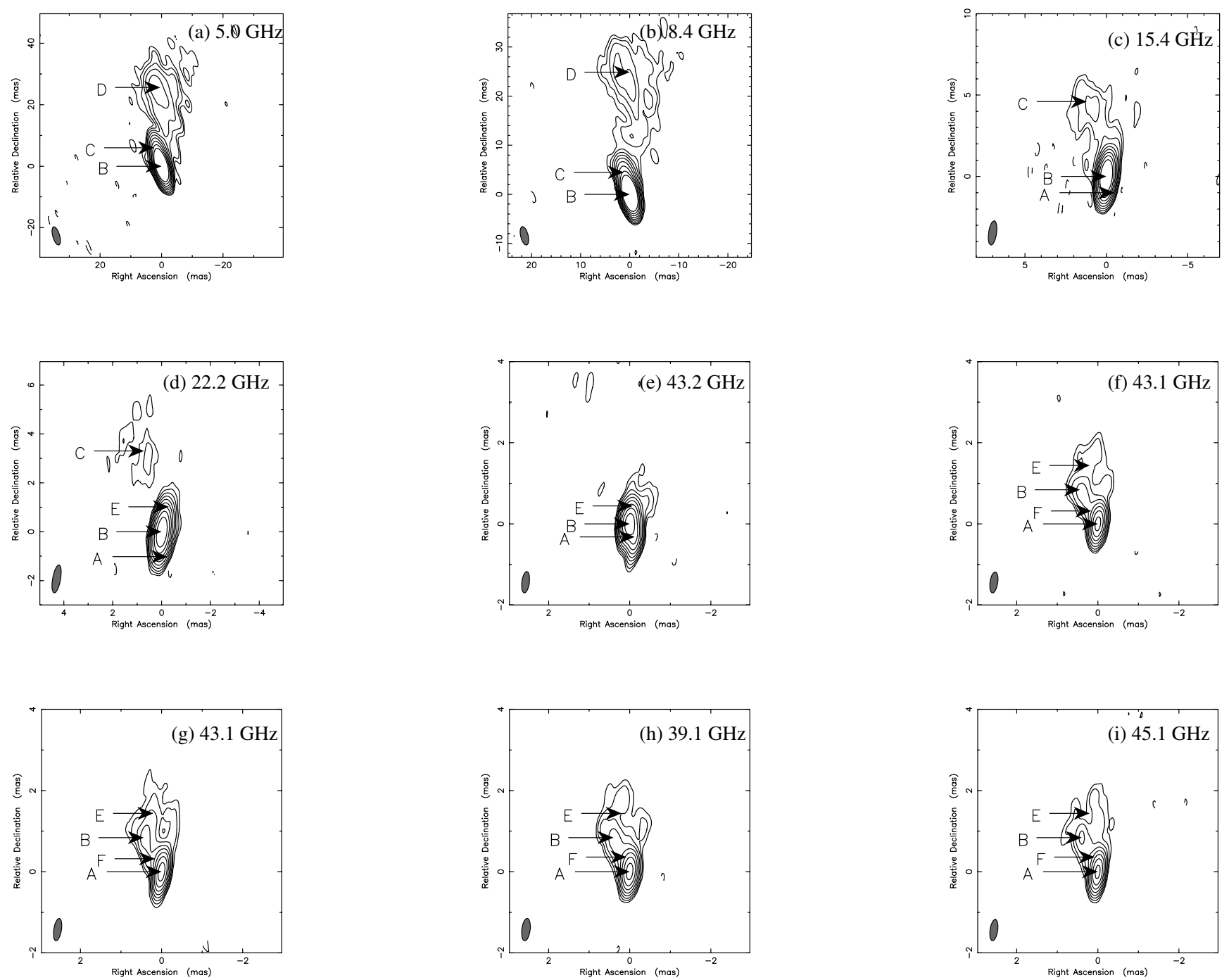

Fig. 2. The clean images of NRAO 530 (detailed parameters are listed in Table 2).

Table 2. Descriptions of VLBI images of NRAO 530.

\begin{tabular}{cccccccc}
\hline \hline & $\begin{array}{c}v \\
(\mathrm{GHz})\end{array}$ & $\begin{array}{c}\text { Epoch } \\
(\text { year }) \\
(3)\end{array}$ & $\begin{array}{c}S_{\text {peak }} \\
(\mathrm{Jy} / \mathrm{beam}) \\
(4)\end{array}$ & $\begin{array}{c}\text { Maj } \\
(\mathrm{mas}) \\
(5)\end{array}$ & $\begin{array}{c}\text { Min } \\
(\mathrm{mas}) \\
(6)\end{array}$ & $\begin{array}{c}\text { PA } \\
(\mathrm{deg}) \\
(7)\end{array}$ & $\begin{array}{c}\text { Contours } \\
(\mathrm{mJy} / \text { beam }) \\
(8)\end{array}$ \\
\hline (a) & 5.0 & 1997.10 & 5.7 & 6.11 & 2.21 & 16.1 & $2.9 \times(-1,1,2,4,8,16,32,64,128,256)$ \\
(b) & 8.4 & 1997.10 & 7.7 & 3.80 & 1.39 & 15.5 & $2.8 \times(-1,1,2,4,8,16,32,64,128,256)$ \\
(c) & 15.4 & 1997.12 & 8.0 & 1.49 & 0.49 & -8.0 & $8.4 \times(-1,1,2,4,8,16,32,64,128,256)$ \\
(d) & 22.2 & 1997.12 & 6.8 & 1.16 & 0.32 & -9.9 & $12.7 \times(-1,1,2,4,8,16,32,64,128,256)$ \\
(e) & 43.2 & 1997.12 & 4.5 & 0.53 & 0.19 & -7.3 & $10.0 \times(-1,1,2,4,8,16,32,64,128,256)$ \\
(f) & 43.1 & 1999.32 & 1.7 & 0.52 & 0.19 & -8.2 & $9.7 \times(-1,1,2,4,8,16,32,64,128)$ \\
(g) & 43.1 & 1999.40 & 1.8 & 0.55 & 0.19 & -9.0 & $9.0 \times(-1,1,2,4,8,16,32,64,128)$ \\
(h) & 39.1 & 1999.42 & 2.0 & 0.55 & 0.20 & -7.0 & $10.8 \times(-1,1,2,4,8,16,32,64,128)$ \\
(i) & 45.1 & 1999.42 & 1.7 & 0.53 & 0.19 & -8.3 & $10.2 \times(-1,1,2,4,8,16,32,64,128)$ \\
\hline
\end{tabular}

Notes: (1) Image index (see Fig. 2); (2) observing frequency; (3) observing epoch; (4) peak flux density; (5), (6), (7) parameters of the restoring Gaussian beam: the full width at half maximum (FWHM) of the major and minor axes and the position angle (PA) of the major axis; (8) contour levels of the image.

The 1997 simultaneous observations at 43,22 , and $15 \mathrm{GHz}$ showed a consistent central core region (Figs. 2c-e), which can be fitted with 3 components, A, B, and E (Table 3 ). The fitted relative separations from $A$ component of 0.35 and 0.53 mas for components $\mathrm{B}$ and $\mathrm{E}$, respectively, at $43 \mathrm{GHz}$ agree reasonably with the results from 22 and $15 \mathrm{GHz}$ considering their $>1$ mas angular resolutions (see Table 2). And it is also possible that the 0.05-0.2 mas difference in the measured core separation for components $\mathrm{B}$ and $\mathrm{E}$ is due to the opacity shift, since the relative separation of the VLBI component from the self-absorbed core is frequency dependent. At $15 \mathrm{GHz}$, component $\mathrm{E}$ seemed too weak to be detected (also refer to Fig. 5 for its spectrum). These 
Table 3. NRAO 530 model parameters.

\begin{tabular}{|c|c|c|c|c|c|c|c|c|c|c|}
\hline (1) & $\begin{array}{c}v \\
(\mathrm{GHz}) \\
(2)\end{array}$ & $\begin{array}{c}\text { Epoch } \\
\text { (year) } \\
(3) \\
\end{array}$ & $\begin{array}{c}\text { Component } \\
(4) \\
\end{array}$ & $\begin{array}{c}S \\
(\mathrm{Jy}) \\
(5) \\
\end{array}$ & $\begin{array}{c}r \\
\text { (mas) } \\
(6) \\
\end{array}$ & $\begin{array}{c}\phi \\
(\mathrm{deg}) \\
(7) \\
\end{array}$ & $\begin{array}{c}\theta_{\text {maj }} \\
(\mathrm{mas}) \\
(8) \\
\end{array}$ & $\begin{array}{c}\theta_{\min } \\
(\mathrm{mas}) \\
(9) \\
\end{array}$ & $\begin{array}{c}\mathrm{PA} \\
(\mathrm{deg}) \\
(10) \\
\end{array}$ & $\begin{array}{c}T_{\mathrm{b}} \\
\left(10^{12} \mathrm{~K}\right) \\
(11) \\
\end{array}$ \\
\hline \multirow[t]{3}{*}{ (a) } & 5.0 & 1997.10 & B & 5.60 & 0 & 0 & 0.78 & 0.23 & 24.3 & 2.9 \\
\hline & & & $\mathrm{C}$ & 0.60 & 4.02 & 14.7 & 3.13 & 1.70 & 7.8 & $1.0 \mathrm{E}-2$ \\
\hline & & & D & 0.53 & 21.92 & 0.4 & 16.30 & 6.12 & 13.2 & $4.9 \mathrm{E}-4$ \\
\hline \multirow[t]{3}{*}{ (b) } & 8.4 & 1997.10 & B & 7.73 & 0 & 0 & 0.35 & 0.21 & 9.0 & 3.5 \\
\hline & & & $\mathrm{C}$ & 0.64 & 3.22 & 12.3 & 4.07 & 1.65 & 20.4 & $3.1 \mathrm{E}-3$ \\
\hline & & & D & 0.33 & 22.89 & 1.1 & 13.60 & 5.42 & 13.9 & $1.5 \mathrm{E}-4$ \\
\hline \multirow[t]{3}{*}{ (c) } & 15.4 & 1997.12 & A & 0.91 & 0 & 0 & 0.21 & 0.21 & $\ldots$ & $2.0 \mathrm{E}-1$ \\
\hline & & & B & 8.01 & 0.38 & 16.5 & 0.22 & 0.10 & 48.6 & 3.66 \\
\hline & & & $\mathrm{C}$ & 0.46 & 3.42 & 10.2 & 4.43 & 1.70 & 22.4 & $5.9 \mathrm{E}-4$ \\
\hline \multirow[t]{4}{*}{ (d) } & 22.2 & 1997.12 & A & 1.87 & 0 & 0 & 0.26 & 0.10 & 12.0 & $3.4 \mathrm{E}-1$ \\
\hline & & & B & 6.73 & 0.29 & 24.5 & 0.21 & 0.16 & 12.3 & $9.4 \mathrm{E}-1$ \\
\hline & & & E & 0.12 & 0.73 & 8.7 & 0.14 & 0.14 & & $2.9 \mathrm{E}-2$ \\
\hline & & & $\mathrm{C}$ & 0.37 & 3.24 & 14.5 & 4.89 & 1.68 & 13.1 & $2.1 \mathrm{E}-4$ \\
\hline \multirow[t]{3}{*}{ (e) } & 43.2 & 1997.12 & A & 1.62 & 0 & 0 & 0.13 & 0.04 & 4.7 & $3.9 \mathrm{E}-1$ \\
\hline & & & B & 5.76 & 0.35 & 21.3 & 0.17 & 0.16 & -42.3 & $2.6 \mathrm{E}-1$ \\
\hline & & & E & 0.58 & 0.53 & 2.4 & 0.66 & 0.28 & 4.3 & $3.9 \mathrm{E}-3$ \\
\hline \multirow[t]{4}{*}{ (f) } & 43.1 & 1999.32 & A & 1.63 & 0 & 0 & 0.08 & 0.05 & -0.3 & $5.1 \mathrm{E}-1$ \\
\hline & & & $\mathrm{F}$ & 0.34 & 0.18 & 13.4 & 0.19 & 0.19 & & $1.2 \mathrm{E}-2$ \\
\hline & & & B & 0.28 & 0.84 & 27.5 & 0.59 & 0.13 & 27.3 & $4.6 \mathrm{E}-3$ \\
\hline & & & E & 0.18 & 1.42 & 2.8 & 1.11 & 0.47 & 17.7 & $4.3 \mathrm{E}-4$ \\
\hline \multirow[t]{4}{*}{ (g) } & 43.1 & 1999.40 & A & 1.86 & 0 & 0 & 0.19 & 0.04 & -12.4 & $3.1 \mathrm{E}-1$ \\
\hline & & & $\mathrm{F}$ & 0.22 & 0.22 & 20.8 & 0.13 & 0.13 & $\ldots$ & $1.6 \mathrm{E}-2$ \\
\hline & & & B & 0.26 & 0.85 & 27.9 & 0.65 & 0.18 & 18.3 & $2.8 \mathrm{E}-3$ \\
\hline & & & E & 0.22 & 1.33 & 2.6 & 1.22 & 0.68 & 19.5 & $3.3 \mathrm{E}-4$ \\
\hline \multirow[t]{4}{*}{ (h) } & 39.1 & 1999.42 & A & 1.78 & 0 & 0 & 0.07 & 0.04 & 10.4 & $9.6 \mathrm{E}-1$ \\
\hline & & & F & 0.56 & 0.17 & 12.2 & 0.34 & 0.09 & 45.5 & $2.8 \mathrm{E}-2$ \\
\hline & & & B & 0.31 & 0.92 & 26.0 & 0.63 & 0.20 & 32.8 & $3.7 \mathrm{E}-3$ \\
\hline & & & $\mathrm{E}$ & 0.21 & 1.48 & 2.8 & 1.24 & 0.54 & 23.6 & $4.8 \mathrm{E}-4$ \\
\hline \multirow[t]{4}{*}{ (i) } & 45.1 & 1999.42 & A & 1.65 & 0 & 0 & 0.12 & 0.02 & -11.2 & $7.8 \mathrm{E}-1$ \\
\hline & & & $\mathrm{F}$ & 0.16 & 0.19 & 18.5 & 0.04 & 0.04 & ; & $1.1 \mathrm{E}-1$ \\
\hline & & & B & 0.34 & 0.71 & 27.4 & 1.21 & 0.12 & 18.3 & $2.7 \mathrm{E}-3$ \\
\hline & & & E & 0.16 & 1.29 & -1.6 & 1.33 & 0.21 & 11.0 & $6.5 \mathrm{E}-4$ \\
\hline
\end{tabular}

Note: (1) Index of image (as shown in Fig. 2); (2) observing frequency; (3) observing epoch; (4) component in each image; (5)-(10) model parameters of the Gaussian component: $S=$ flux density, $r=$ angular separation, and $\phi=$ position angle of component with respect to the origin $(0,0)$, full widths at half maximum $(F W H M)$ of the major $\left(\theta_{\text {maj }}\right)$ and minor $\left(\theta_{\min }\right)$ axes and the position angle $(\mathrm{PA})$ of the major axis; (11) brightness temperature.

are comparable to the results obtained from four $43 \mathrm{GHz}$ images at epochs 1995.79 to 1996.90 (Jorstad et al. 2001) and an $86 \mathrm{GHz}$ image at epoch 1995.38 (Bower et al. 1997). Another four $43 \mathrm{GHz}$ images (Figs. 2f-i) made in April-May 1999 can be well fitted with 4 components: components $\mathrm{A}, \mathrm{B}, \mathrm{E}$, and a new component $\mathrm{F}$ at 0.18 mas with a PA of $25^{\circ}$.

By combining our new measurements of angular separations with the published data in 1995 and 1996 (Jorstad et al. 2001) of components $\mathrm{B}$ and $\mathrm{E}$, we obtained proper motions of $0.21 \pm 0.02$

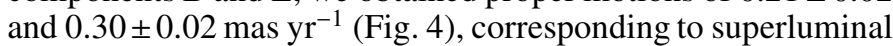
velocities of $10.2 \pm 0.8 \mathrm{c}$ and $14.5 \pm 1.1 \mathrm{c}$ for components $\mathrm{B}$ and $\mathrm{E}$, respectively. This implies that the ejecting times for components $\mathrm{B}$ and $\mathrm{E}$ are around 1995.46 and 1994.76, respectively. These two epochs fall just inside the outburst phase shown in Fig. 1 (also see discussion in Sect. 3.3). EGRET reported the increasing $\gamma$-ray flux densities from the direction of NRAO 530 during two periods (1994.2-1994.6 and 1995.4-1995.5) with the average fluxes of $64 \pm 26 \mathrm{pJy}$ and $127 \pm 48 \mathrm{pJy}$ (Hartman et al. 1999). As EGRET could not observe NRAO 530 with dense time sampling and long integrations, the $\gamma$-ray measurements have large uncertainties and cannot rule out that $\gamma$-ray flares were missed or that a maximum of an ongoing flare was really seen. According to the existing data (VLBA and EGRET) on NRAO 530, we think that the emergence of components $\mathrm{B}$ and $\mathrm{E}$ are closely following the $\gamma$-ray flares in NRAO 530. The activity of $\gamma$-ray has been found to be correlated with infrared to X-ray activity in 3C 279 (Maraschi et al. 1994) and to occur before or during a radio and millimeter outburst in many sources (Reich et al. 1993).

For component $\mathrm{C}$, we also plotted its separation from the core (Fig. 4) as a function of time measured in 1992, 1995, 1996, and 1997 by Shen et al. (1997), Jorstad et al. (2001), Bower \& Backer (1998), and us in this work, respectively. The best-fitted

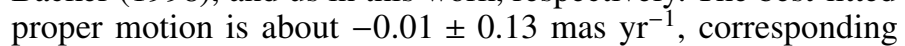
to an apparent velocity of $-0.35 \pm 6.04 \mathrm{c}$. This is quite different from the apparent velocity of $29 \pm 9 h^{-1} \mathrm{c}$ (Hubble constant $H_{0}=100 \mathrm{hm} \mathrm{s}^{-1} \mathrm{Mpc}^{-1}$, deceleration parameter $q_{0}=0.1$, and cosmological constant $\Lambda=0$ ) reported by Jorstad et al. (2001) (component $\mathrm{D}$ in their paper). Our new estimate with more time coverage in the data indicates that there is no appreciable motion for component $\mathrm{C}$. The previously reported high apparent velocity of $29 \pm 9 h^{-1} \mathrm{c}$ may be due to their limited epochs of data and thus the large uncertainty. This discrepancy may be related to the complex structure in component $\mathrm{C}$ (Figs. 2c and d), which results in a large error in the determination of its position. This kind of diffused morphology could be due to internal jet structure, blending effects of shocks, or some sort of interaction. For 

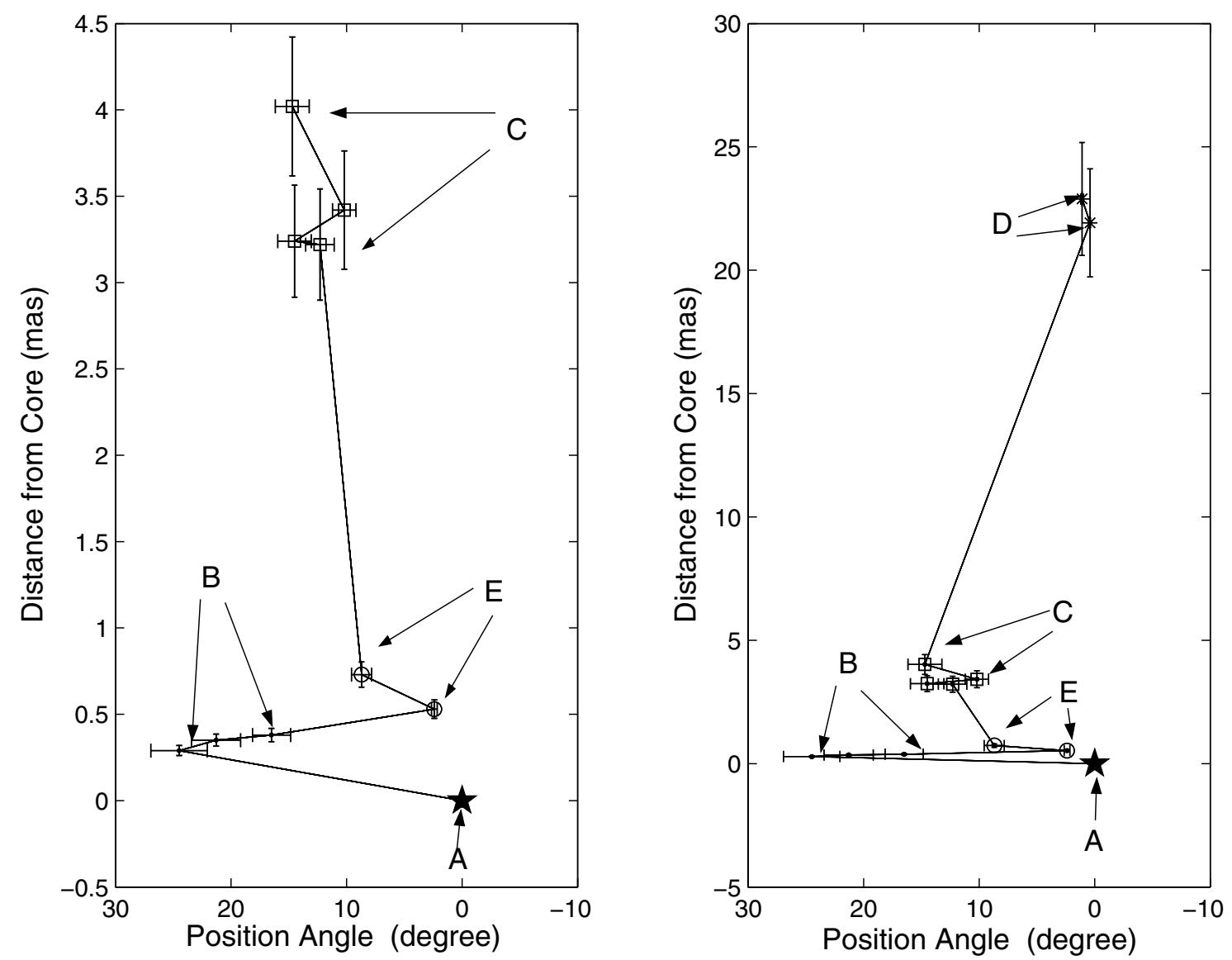

Fig. 3. The projected trajectory of jet components observed in 1997: (left) within central 4 mas, (right) within 20 mas, showing a continuously bending jet moving away from the central core. A, B, E, C, and D represent different VLBI components and the solid lines are connections of individual jet components.

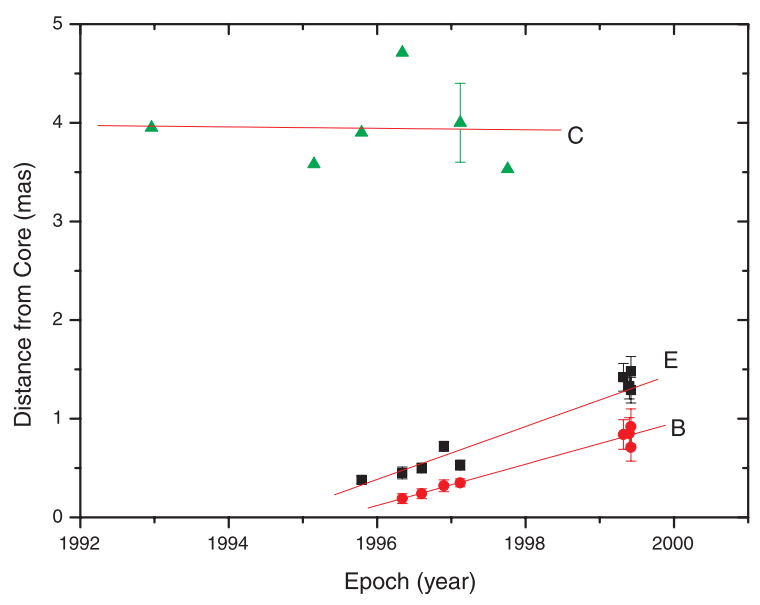

Fig. 4. Separation of components B, E, and C from the assumed core component $\mathrm{A}$ as the function of the observing epoch.

the reason to be discussed in Sect. 4, we think it is mainly caused by the interaction of jet emission with the surrounding medium.

\subsection{Components' spectra}

For the quasi-simultaneous observations in February 1997, the spectra of VLBI components (A, B, C, and D) can be fit with $S_{v} \propto v^{-\alpha}$, and the resultant optically thin spectral indexes are $\alpha_{\mathrm{A}}=0.21, \alpha_{\mathrm{B}}=0.31 \pm 0.07, \alpha_{\mathrm{C}}=0.58 \pm 0.01$, and $\alpha_{\mathrm{D}}=0.91$.
Table 4. Spectral fitting results with the SSA and FFA.

\begin{tabular}{|c|c|c|c|c|c|c|}
\hline \multirow[t]{2}{*}{ Parameters } & \multicolumn{3}{|c|}{ SSA } & \multicolumn{2}{|c|}{ FFA } & \multirow[b]{2}{*}{$\mathrm{A}+\mathrm{B}$} \\
\hline & $\mathrm{B}$ & $\mathrm{C}$ & $\mathrm{A}+\mathrm{B}$ & $\mathrm{B}$ & $\mathrm{C}$ & \\
\hline$\alpha$ & 0.31 & 0.64 & 0.14 & 0.35 & 0.79 & 0.19 \\
\hline$S_{0}(\mathrm{Jy})$ & 0.12 & 0.02 & 0.13 & 21.77 & 4.38 & 15.59 \\
\hline$\tau$ & 148.31 & 159.90 & 101.40 & 23.33 & 20.88 & 21.89 \\
\hline
\end{tabular}

Component A is regarded as the core of NRAO 530 since it has the flattest spectral index and it is at the south end of the overall emission. Component E seems to show an inverted spectrum with a high turnover frequency (greater than $43 \mathrm{GHz}$ ) in 1997. This is likely to be due to the strong variability at $43 \mathrm{GHz}$, as 1999 data had a three times lower flux density. The fitted twopoint spectral index is $\alpha_{\mathrm{E}}=-1.5$ between 22 and $43 \mathrm{GHz}$.

In order to discuss the possibly dominant mechanism for the observed low-frequency absorption in components B and C, both the synchrotron self-absorption (SSA) and the free-free absorption (FFA) are tried to fit the convex spectra with the following formulae,

$S_{v}=S_{0} v^{2.5}\left[1-\exp \left(-\tau_{\mathrm{s}} v^{-\alpha-2.5}\right)\right]$

for the SSA and

$S_{v}=S_{0} v^{-\alpha} \exp \left(-\tau_{\mathrm{f}} v^{-2.1}\right)$

for the FFA, where $v$ is the observing frequency in $\mathrm{GHz}, S_{0}$ the intrinsic flux density at $1 \mathrm{GHz}$ in $\mathrm{Jy}, \tau_{\mathrm{f}}$ and $\tau_{\mathrm{s}}$ are the SSA and 

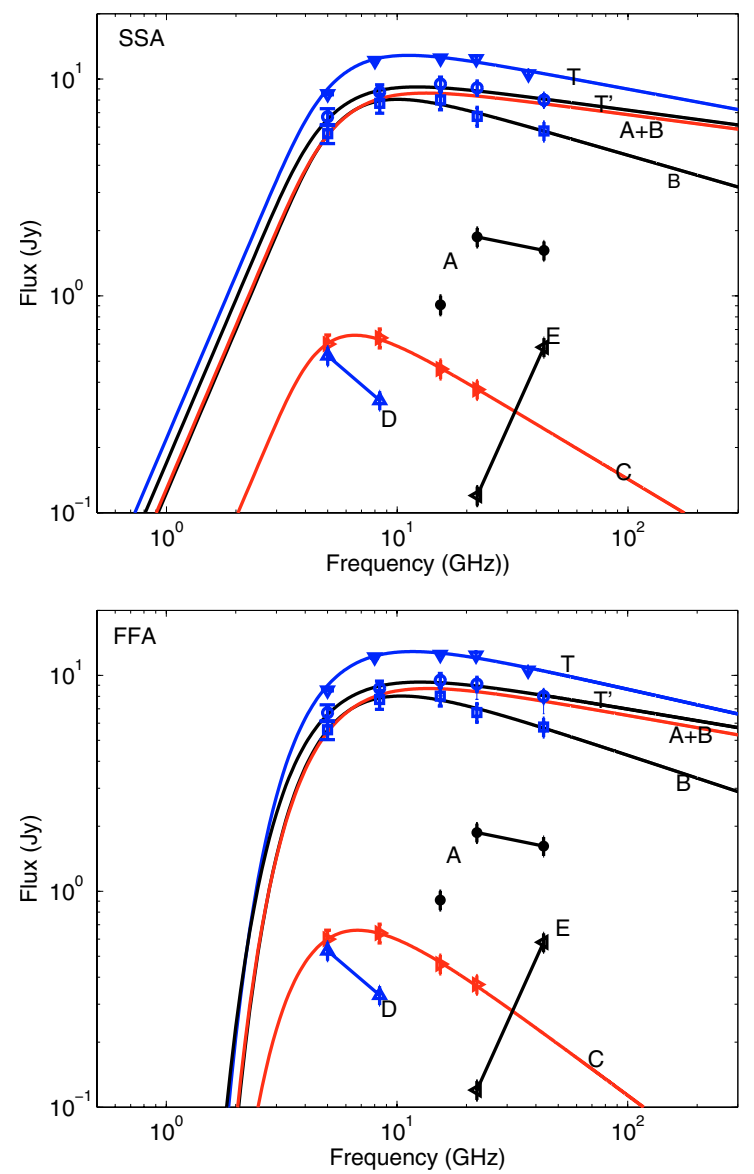

Fig. 5. Component spectral fit of NRAO 530 in February 1997 (top: spectra are fit with the SSA, bottom: spectra are fit with the FFA.

FFA coefficients at $1 \mathrm{GHz}$, respectively. We also performed the fits to the combined component $(\mathrm{A}+\mathrm{B})$ emission, which is believed to be represented by component $\mathrm{B}$ at low frequencies $(5$ and $8 \mathrm{GHz}$ ) due to the limited resolutions (see Table 3) and is resolved out at frequencies $\geq 15 \mathrm{GHz}$. All the fitting results are listed in Table 4 with the fitting curves shown in Fig. 5.

Both the SSA and FFA fit the spectra of components B and $\mathrm{C}$ equally well. Thus, the spectral fit itself is not adequate for telling which mechanism is mainly responsible for the absorption towards components B and C. However, a short synchrotron cooling timescale seems to suggest that FFA plays an important role in component $\mathrm{C}$ (see Sect. 4). If so, that the FFA coefficients for components B and C are almost the same (see Table 4) implies the presence of the FFA by the same surrounding medium in component $\mathrm{B}$. Then, one could estimate that the absorbing material should cover at least $25 \mathrm{pc}$ in size to produce the same FFA towards components $\mathrm{B}$ and $\mathrm{C}$, which were separated by about 3 mas in the sky in 1997. The interaction of the surrounding interstellar medium causing FFA with the jet emission in NRAO 530 can be used to explain the poorly determined proper motion in component $\mathrm{C}$ (see Sect. 3.1).

For comparison, we also plotted the spectrum of the sum of all the VLBI components (labelled by $\mathrm{T}^{\prime}$ in Fig. 5), as well as the spectrum from single-dish data (labelled by T in Fig. 5). The missing of flux density is persistently seen in all five VLBI frequencies. This is probably due to the effect of the resolution of the extended components. Therefore our amplitude calibrations, including the atmospheric opacity corrections, should be reasonable.

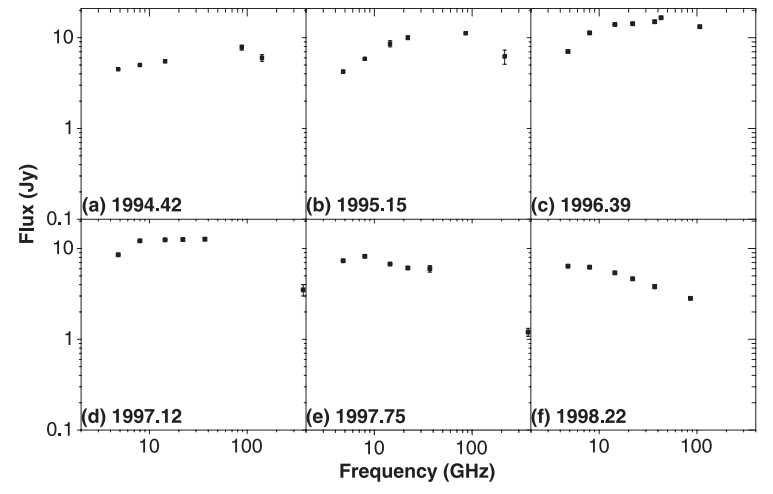

Fig. 6. Spectral variability seen in a flare from 1994.5 to 1998.5 . Flux densities at 43, 86, 88, 95, 107, 142, 150, 215, and $375 \mathrm{GHz}$ are listed in (Table 5). Letters a, b, c, d, e, f represent different epochs indicated in Fig. 1.

Table 5. Total flux density data of NRAO 530 at $v \geq 43 \mathrm{GHz}$.

\begin{tabular}{ccccl}
\hline \hline $\begin{array}{c}v \\
(\mathrm{GHz})\end{array}$ & $\begin{array}{c}\text { Epoch } \\
(\text { Year })\end{array}$ & $\begin{array}{c}\text { Flux } \\
(\mathrm{Jy})\end{array}$ & $\begin{array}{c}\text { Error } \\
(\mathrm{Jy})\end{array}$ & References \\
\hline 43 & 1996.39 & 16.6 & 0.5 & Falcke et al. (1998) \\
86 & 1995.18 & 11.2 & 0.3 & Krichbaum et al. (1997) \\
88 & 1994.42 & 7.8 & 0.5 & Reuter et al. (1997) \\
95 & 1996.82 & 12.6 & 0.7 & Falcke et al. (1998) \\
107 & 1996.39 & 13.2 & 0.4 & Falcke et al. (1998) \\
142 & 1994.42 & 6.0 & 0.5 & Reuter et al. (1997) \\
150 & 1996.82 & 12.0 & 1.2 & Falcke et al. (1998) \\
215 & 1995.18 & 6.2 & 1.1 & Krichbaum et al. (1997) \\
375 & 1997.12 & 3.5 & 0.5 & Robson et al. (2001) \\
375 & 1997.26 & 2.7 & 0.3 & Robson et al. (2001) \\
375 & 1997.75 & 1.2 & 0.1 & Robson et al. (2001) \\
\hline
\end{tabular}

\subsection{Spectral variability}

A complete outburst profile can be traced from mid-1994 to mid-1998 Fig. 1. To analyze spectral variability during this radio outburst we selected six epochs: 1994.42 (a), 1995.18 (b), 1996.39 (c), 1997.12 (d), 1997.75 (e), and 1998.22 (f). Figure 6 shows the spectral distribution at these six epochs based on the data points in Fig. 1 (at frequencies $\leq 37.0 \mathrm{GHz}$ ) and in Table 5 (at frequencies $\geq 43 \mathrm{GHz}$ ). It is quite clear in Fig. 6 that the turnover frequency increases during the rising phase (represented by epochs (a), (b), and (c)) when the flux density is increasing (see Fig. 1).

From 1997.12 (d) the turnover frequency starts to decrease when the total flux density decreases. This is because high frequency photons lose energy quickly with jet expansion and thus the flux density at high frequency decreases, too. In 1998.22 (f) the spectrum returns to the level of quiescent phase.

\section{Discussion}

It is well known that the observed transverse velocity $\left(\beta_{\text {app }}=\right.$ $v_{\text {app }} / c, v_{\text {app }}$ the apparent velocity) is related to the true velocity $(\beta)$ and the angle to the line of sight $(\theta)$ by $\beta_{\text {app }}=\beta \sin \theta /(1-$ $\beta \cos \theta$ ). Therefore, the maximum angle $(\theta)$ of $11.2^{\circ}$ and $7.9^{\circ}$ for components $\mathrm{B}$ and $\mathrm{E}$ can be estimated from their corresponding apparent velocities of $10.2 \mathrm{c}$ and $14.5 \mathrm{c}$.

The Doppler factor $(\delta)$ is defined as $\delta=\Gamma^{-1}(1-\beta \cos \theta)^{-1}$, where $\Gamma=\left(1-\beta^{2}\right)^{-1 / 2}$ is the Lorentz factor. By means of the above expression of $\beta_{\text {app }}$, we can derive $\delta=\Gamma \pm\left(\Gamma^{2}-\beta_{\text {app }}^{2}-1\right)^{1 / 2}$. Then the Doppler factor of component B is $\delta=\Gamma_{\min }=10.2$ 
when the Lorentz factor is $\Gamma_{\min }=\left(1+\beta_{\mathrm{app}}^{2}\right)^{1 / 2}$. In the same way we compute the Doppler factor of another superluminal jet component $\mathrm{E}, \delta=14.5$.

For another scenario in which no intrinsic acceleration or deceleration happened in the jet motion, i.e., jets are ejected with the same velocity $\beta$ (or a constant Lorentz factor $\Gamma$ ), the changes of apparent velocities would be mainly due to different viewing angles (variable $\theta$ ). The fastest apparent speed of $14.5 \mathrm{c}$ (for component E) observed in NRAO 530 will limit the constant Lorentz factor to at least of 14.5 , so we use $\Gamma_{\min }=15$. Combining with the apparent velocity measurements, we can obtain the viewing angles of $9.7^{\circ}$ and $1.6^{\circ}$ for component $\mathrm{B}$ with $\beta_{\text {app }}=10.2 \mathrm{c}$, and of $4.9^{\circ}$ and $3.0^{\circ}$ for component $\mathrm{E}$ with $\beta_{\text {app }}=14.5 \mathrm{c}$. The corresponding Doppler factors are 4.0 and 26.0 for component $\mathrm{B}$, and 11.3 and 18.7 for component $\mathrm{E}$.

Estimates of the magnetic field in a radio source can be obtained in two distinct ways. First, by assuming the equipartition of energy between the particles and the magnetic field, one can have (e.g., Pacholczyk 1970)

$B^{\mathrm{eq}}=\left[4.5(1+k) f\left(\alpha, v_{1}, v_{2}\right) L R^{-3}\right]^{2 / 7}$,

where $B^{\text {eq }}$ in $\mathrm{G}$ is the magnetic field when the total energy of magnetic field and particles has a minimum value (equipartition between the energy of the particles and the energy of the magnetic field), $k$ is the energy ratio between the heavy particles and the electrons (we used $k=100), f\left(\alpha, v_{1}, v_{2}\right)$ is tabulated in Appendix 2 of Pacholczyk (1970), $\alpha$ is the optically thin spectral index, $v_{1}$ and $v_{2}$ are the lower and upper cutoff frequencies of the synchrotron spectrum with typical values of $10^{7}$ and $10^{11} \mathrm{~Hz}, R=\frac{d_{L} \Theta}{2(1+z)}$ is the radius of component in $\mathrm{cm}$ (here, $\Theta$ is the angular diameter and $d_{L}$ is luminosity distance), and $L$ is the synchrotron luminosity in erg $\mathrm{s}^{-1}$ and can be expressed as $L=4 \pi d_{L}^{2} \int_{v_{1}}^{v_{2}} S_{\nu} \mathrm{d} v$ (here, $S_{v}$ is the flux density at a frequency $v)$. It should be mentioned that $B^{\text {eq }}$ is insensitive to the values of $k$ and the fraction of the source's volume occupied by the magnetic field and the particles, which is assumed to be 1 . By assuming $\int_{v_{1}}^{v_{2}} S_{v} \mathrm{~d} v \simeq S_{\mathrm{m}} v_{\mathrm{m}}$, we can rewrite

$B^{\mathrm{eq}} \simeq 4.0 \times 10^{-4}\left[f\left(\alpha, 10^{7}, 10^{11}\right)(1+z)^{3} d_{L}^{-1} \Theta^{-3} S_{\mathrm{m}} \nu_{\mathrm{m}}\right]^{2 / 7}$,

where $v_{\mathrm{m}}$ is the turnover frequency in $\mathrm{GHz}, S_{\mathrm{m}}$ the extrapolated optically thin flux density at the turnover frequency in Jy, and luminosity distance $d_{L}$ and angular diameter $\Theta$ are in Gpc and mas, respectively.

Secondly, assuming that the inverted spectrum is due to SSA, one can estimate the magnetic field (i.e., Marscher 1983),

$B^{\text {syn }}=10^{-5} b(\alpha) \Theta^{4} v_{\mathrm{m}}^{5} S_{\mathrm{m}}^{-2} \delta /(1+z)$

where $B^{\text {syn }}$ is the magnetic field in $\mathrm{G}$, and $b(\alpha)$ is a tabulated parameter dependent on the spectral index $\alpha$ (Table 1 in Marscher 1983 ). For component $\mathrm{B}, \alpha=0.31$ gives $b(0.31) \approx 3.0$.

From the SSA spectral-fitting results for component $\mathrm{B}(\Theta=$ $\sqrt{\theta_{\text {maj }} \times \theta_{\text {min }}}=0.2$ mas, $\left.S_{\mathrm{m}}=8 \mathrm{Jy}, v_{m}=10 \mathrm{GHz}\right)$ and $\delta=1$ (without consideration of relativistically beaming effect), we obtained $B_{\mathrm{B}}^{\mathrm{eq}} \simeq 580 \mathrm{mG}$ and $B_{\mathrm{B}}^{\mathrm{syn}} \simeq 4 \times 10^{-2} \mathrm{mG}$. The time scale for an electron to lose its energy through synchrotron radiation is $t_{1 / 2} \simeq 2.76 \times 10^{4}(\mathrm{yr})\left(\frac{B^{\text {syn }}}{1 \mathrm{mG}}\right)^{-1.5}\left(\frac{v_{\mathrm{m}}}{1 \mathrm{GHz}}\right)^{-0.5}$. This gives a cooling time scale of $1.1 \times 10^{6} \mathrm{yr}$ for component $\mathrm{B}$, while the intrinsic cooling time scale in the source rest frame should be greater when the Doppler factor is larger than $(1+z)$. In the same way, we computed the magnetic field for component $\mathrm{A}, B_{\mathrm{A}}^{\mathrm{eq}} \simeq 560 \mathrm{mG}$, and $B_{\mathrm{A}}^{\mathrm{syn}} \simeq 8 \mathrm{mG}$ with $S_{\mathrm{m}}=1.87 \mathrm{Jy}, v_{\mathrm{m}}=22.2 \mathrm{GHz}$,
$\Theta=0.16$ mas, $\alpha=0.21$, and $\delta=1$, since it seems to have a (presumably synchrotron self-absorbed) inverted spectrum at frequencies between 15 and $22 \mathrm{GHz}$ (see Fig. 5).

The ratio of the particle energy density $\left(u_{\mathrm{p}}\right)$ to the energy density of the magnetic field $\left(u_{\mathrm{m}}\right)$ can be expressed as (i.e., Readhead 1994) $\frac{u_{\mathrm{p}}}{u_{\mathrm{m}}}=\left(\frac{B^{\mathrm{eq}}}{B^{\mathrm{syn}}}\right)^{17 / 4}$. We get $\frac{u_{\mathrm{p}}}{u_{\mathrm{m}}} \simeq 6.9 \times 10^{7}$ and $\frac{u_{\mathrm{p}}}{u_{\mathrm{m}}} \simeq 5.5 \times 10^{17}$ for components $\mathrm{A}$ and $\mathrm{B}$, implying that both components A and B are particle-dominated or that their emissions are relativistically beamed. In the former case, for component B this high ratio may account for the observed fast decrease in the radio flux density (from $5.76 \mathrm{Jy}$ to $\sim 0.3 \mathrm{Jy}$ ) in two years even though its synchrotron cooling time could be much longer $\left(\simeq 1.1 \times 10^{6} \mathrm{yr}\right)$. This is because the high ratio of particle to magnetic field energies may cause the inverse Compton catastrophe (Readhead 1994), and inverse Compton scattering of X-ray photons may further result in the $\gamma$-ray emission (e.g., Hartman et al. 1992).

In the latter case, as the equations for the magnetic field (Eqs. (5) and (6)) have different dependencies on the Doppler boosting factor, we thus can obtain

$$
\frac{B^{\mathrm{eq}}}{B^{\mathrm{syn}}} \simeq\left(\frac{B_{\mathrm{int}}^{\mathrm{eq}}}{B_{\mathrm{int}}^{\mathrm{syn}}}\right)\left(\frac{\delta}{1+z}\right)^{6+\frac{16 \alpha}{7}}
$$

where $B^{\text {eq }}$ and $B^{\text {syn }}$ are the measured magnetic field, and $B_{\text {int }}^{\text {eq }}$ and $B_{\text {int }}^{\text {syn }}$ are the magnetic field estimated in the source rest frame. When $B_{\text {int }}^{\mathrm{eq}} \simeq B_{\mathrm{int}}^{\mathrm{syn}}$, the Doppler factor in Eq. (7) is defined as the equipartition Doppler-factor, $\delta^{\mathrm{eq}}$. To derive Eq. (7), we used the following relations, $v_{\mathrm{m}}^{\mathrm{ob}}=\left(\frac{\delta^{\mathrm{eq}}}{1+z}\right) v_{\mathrm{m}}^{\prime}, S_{\mathrm{m}}^{\mathrm{ob}}=\left(\frac{\delta^{\mathrm{eq}}}{1+z}\right)^{3+\alpha} S_{\mathrm{m}}^{\prime}$, and $\Theta^{\mathrm{ob}}=$ $\left(\frac{1+z}{\delta^{\text {eq }}}\right) \Theta^{\prime}$ (Singal \& Gopal-Krishna 1985), ahere $\Theta^{\mathrm{ob}}, v_{\mathrm{m}}^{\mathrm{ob}}$, and $S_{\mathrm{m}}^{\mathrm{ob}}$ are angular diameter, inverted frequency, and corresponding flux density in the observer's frame, respectively, and $\Theta^{\prime}, v_{\mathrm{m}}^{\prime}$, and $S_{\mathrm{m}}^{\prime}$ in the source rest frame. Then we got the equipartition Dopplerfactors for components $\mathrm{A}$ and $\mathrm{B}$ of $\delta_{\mathrm{A}}^{\mathrm{eq}} \simeq 3.7$ and $\delta_{\mathrm{B}}^{\mathrm{eq}} \simeq 7.2$, respectively. These are comparable to the equipartition Doppler factors of 7.0 at $10 \mathrm{GHz}$ (Marscher et al. 1979) and 5.2 at 15 GHz (Güijosa \& Daly 1996).

Similarly, for component $C$ we estimated $B_{\mathrm{C}}^{\mathrm{eq}} \simeq 36 \mathrm{mG}$, $B_{\mathrm{C}}^{\mathrm{syn}} \simeq 29 \times 10^{3} \mathrm{mG}, u_{\mathrm{p}} / u_{\mathrm{m}} \simeq 7.4 \times 10^{-13}, \delta_{\mathrm{C}}^{\mathrm{eq}} \simeq 0.8$, and the synchrotron cooling time of $\sim 0.8$ day, with the following parameters: $S_{\mathrm{m}}=0.65 \mathrm{Jy}, v_{m}=7 \mathrm{GHz}, \Theta=2.5 \mathrm{mas}, \alpha=0.53$, and $\delta=1.0$. As the magnetic field is strongly dependent on measured component parameters, the high magnetic field of component $\mathrm{C}$ may be due to its large size ( $\sim 2.5$ mas $)$. Assuming that the equipartition is maintained in component $\mathrm{C}$, we can estimate a size scale of 0.64 mas for component $C$ so that both the calculated $B_{\mathrm{C}}^{\mathrm{eq}}$ and $B_{\mathrm{C}}^{\mathrm{syn}}$ are equal to $120 \mathrm{mG}$. The resulting synchrotron cooling time would be $7.6 \mathrm{yr}$, still too short for the SSA model, so it is very likely that the FFA of the surrounding medium dominates the observed absorption in the component although we cannot completely rule out the SSA effect.

As the Doppler-factors of jets are expected to increase with decreasing viewing angle for the assumed constant Lorentz factor, the equipartition Doppler-factors also should decrease systematically along a bent jet given that the viewing angle increases monotonically along the bending trajectory. This is probably the reason that the $\delta_{\text {eq }}$ of component $\mathrm{C}$ is smaller than the values of components $\mathrm{A}$ and $\mathrm{B}$. However, the fact that $\delta_{\mathrm{B}}^{\mathrm{eq}}>\delta_{\mathrm{A}}^{\mathrm{eq}}$ is consistent with component $\mathrm{B}$ being brighter than component A in 1997. For component B, the Doppler-factor estimated from $\beta_{\text {app }}$ of $\delta=\Gamma_{\min }=10.2$ is comparable to the equipartition Doppler-factor $\delta_{\mathrm{B}}^{\mathrm{eq}} \simeq 7.2$, suggesting that jets most 
likely have the minimum Lorentz factors in an equivalent state. For component $\mathrm{C}$, there is no appreciable proper motion, which suggests a Doppler-factor of $\sim 1.0\left(\delta=\Gamma_{\min }=\sqrt{1+\beta_{\mathrm{app}}^{2}}\right)$. This is consistent with the estimated equipartition Doppler-factor of 0.8 , inferring that it approaches an energy equivalent state between particle and magnetic field in the source rest frame. The fact that $\delta_{\mathrm{C}}^{\mathrm{eq}} \simeq 0.8$ is smaller than $1+z=1.902$ for component $\mathrm{C}$ in NRAO 530 implies that it is Hubble flow that results in the observed departure from the energy equipartition in component $\mathrm{C}$.

\section{Summary}

We have presented the high-resolution VLBI images of NRAO 530 made at 5, 8, 15, 22, 39, 43, $45 \mathrm{GHz}$ in 1997 and 1999. By combining our new measurements with the past data, we estimated superluminal motions of components B and E at apparent velocities of $10.2 \mathrm{c}$ and $14.5 \mathrm{c}$, respectively. These are consistent with components B and E being ejected in 1995.46 and 1994.76 following the $\gamma$-ray flares of 127 and 64 pJy, respectively. Flat spectra are seen in the two central components (A and $\mathrm{B}$ ), and the most compact component $\mathrm{A}$ with the flattest spectral index at the south end is identified as the core. We derived the equipartition Doppler-factor based on Eq. (5) and (6), and obtained $\delta^{\mathrm{eq}} \mathrm{S}$ of 3.7, 7.2, and 0.8 for components $\mathrm{A}, \mathrm{B}$, and $\mathrm{C}$, respectively. These are consistent with a higher flux density in component $\mathrm{B}$, the non-detection of proper motion in component $\mathrm{C}$, and a bent jet. The synchrotron cooling timescale argues for the FFA being responsible for the inverted spectrum of component $\mathrm{C}$, although the SSA effect cannot be completely ruled out yet. While both the SSA and FFA could play a role in component B, we propose that the FFA may be at work given that almost the same FFA coefficients were fitted for both components B and C. If so, the absorbing material would have a projected size of at least $25 \mathrm{pc}$.
Acknowledgements. This research made use of data from the University of Michigan Radio Astronomy Observatory, supported by the University of Michigan and the National Science Foundation. We thank the anonymous referee for helpful comments and suggestions. This work is supported in part by the National Natural Science Foundation of China under grant 10573029. Z.-Q. Shen acknowledges the support by the One-Hundred-Talent Program of Chinese Academy of Sciences.

\section{References}

Aller, H. D., Aller, M. F., Latimer, G. E., \& Hodge, P. E. 1985, ApJS, 59, 513 Bondi, M., Padrielli, L., Fanti, R., et al. 1996, A\&A, 308, 415

Bower, G. C., \& Backer, D. C. 1998, ApJ, 507, 117

Bower, G. C., Backer, D. C., Wright, M., \& Forster, J. R. 1997, ApJ, 484, 118 Falcke, H., Goss, W. M., Matsuo, H., Teuben, P., \& Zhao, J- H. 1998, ApJ, 499, 731

Güijosa, A., \& Daly, R. A. 1996, ApJ, 461, 600

Hartman, R. C., Bertsch, D. L., Fichtel, C. E., et al. 1992, ApJ, 385, 1

Hartman, R. C., Bertsch, D. L., Bloom, S. D., et al. 1999, ApJS, 123, 79

Jorstad, S. G., Marscher, A. P., Mattox, J. R., et al. 2001, ApJS, 134, 181

Junkkarinen, V. 1984, PASP, 96, 539

Krichbaum, T. P., Graham, D. A., Greve, A., et al. 1997, A\&A, 323, 17

Maraschi, L., Grandi, P., Urry, C. M., et al. 1994, ApJ, 435, 91

Marscher, A. P. 1983, ApJ, 264, 296

Marscher, A. P., Marshall, F. E., Mushotzky, R. F., et al. 1979, ApJ, 233, 498

Marscher, A. P., \& Broderick, J. J. 1981, ApJ, 249, 406

Medd, W. J., Andrew, B. H., Harvey, G. A., \& Locke, J. L. 1972, MNRAS, 77, 109

Pacholczyk, A. G. 1970, Radio astrophysics (San Francisco: Freeman), 171

Pollock, J. T., Pica, A. J., Smith, A. G., et al. 1979, AJ, 84, 1658

Readhead, A. C. 1994, ApJ, 624, 51

Reich, W., Steppe, H., Schlickeiser, R., et al. 1993, A\&A, 273, 65

Reuter, H. P., Kramer, C., Sievers, A., et al. 1997, A\&AS, 122, 271

Robson, E. I., Stevens, J. A., \& Jenness, T. 2001, MNRAS, 327, 751

Shen, Z.-Q., Wan, T.-S., Moran, J. M., et al. 1997, AJ, 114, 1199

Singal, A. K., \& Gopal-Krishan 1985, MNRAS, 215, 383

Teräsranta, H., Achren, J., Hanski, M., et al. 2004, A\&A, 427, 769

Tingay, S. J., Murphy, D. W., \& Edwards, P. G. 1998 ApJ, 500, 673

Welch, W. J., \& Spinrad, H. 1973, PASP, 85, 456 